IOS Press

\title{
Treatment with nerve grafts and aFGF attenuates allodynia caused by cervical root transection injuries
}

\author{
Yi-Lo Lin ${ }^{\mathrm{a}, \mathrm{g}}$, Huai-Sheng Kuo ${ }^{\mathrm{a}}$, Ming-Jei Lo ${ }^{\mathrm{a}}$, May-Jywan Tsai ${ }^{\mathrm{a}}$, Meng-Jen Lee, ${ }^{\mathrm{a}, \mathrm{h}}$, Wen-Cheng \\ Huang ${ }^{\mathrm{a}, \mathrm{b}}$, Wen-Chun Kuo ${ }^{\mathrm{a}}$, Yang-Hsin Shih ${ }^{\mathrm{c}}$, Henrich Cheng ${ }^{\mathrm{a}, \mathrm{b}, \mathrm{d}, \mathrm{e}, 1}$ and Ming-Chao Huang ${ }^{\mathrm{a}, \mathrm{b}, \mathrm{f}, 1, *}$ \\ ${ }^{a}$ Neural Regeneration Laboratory, Department of Neurosurgery, Neurological Institute, Taipei Veterans \\ General Hospital, Taipei, Taiwan \\ ${ }^{\mathrm{b}}$ Center for Neural Regeneration, Department of Neurosurgery, Neurological Institute, Taipei Veterans \\ General Hospital, Taipei, Taiwan \\ ${ }^{\mathrm{c}}$ Department of Neurosurgery, Neurological Institute, Taipei Veterans General Hospital, \\ Taipei, Taiwan \\ ${ }^{\mathrm{d}}$ Department \& Institute of Pharmacology, School of Medicine, National Yang-Ming University, Taipei, Taiwan \\ ${ }^{\mathrm{e}}$ Brain Research Center, School of Medicine, National Yang-Ming University, Taipei, Taiwan \\ ${ }^{\mathrm{f}}$ School of Medicine, Taipei Medical University, Taipei, Taiwan \\ ${ }^{\mathrm{g}}$ College of Veterinary Medicine, National Chung Hsing University, Taichung, Taiwan \\ ${ }^{\mathrm{h}}$ Institute of Biochemical Sciences and Technology, Chaoyang University of Technology, Taichung, Taiwan
}

\begin{abstract}
Purpose: Nerve root traction injuries induce spinal cord inflammation and lead to neuronal death within days. In the present study, we examined the inflammatory response one week after multiple cervical root transections.

Methods: In the transection group, the left cervical roots (C6-8) of rats were cut at the spinal cord junction. In the repair group, transected roots were repaired with nerve grafts and the subsequent application of aFGF and fibrin glue. A sham group had nerve roots exposed without transection. Mechanical allodynia and spinal glial responses were evaluated.

Results: Allodynia did not differ between the treatment groups on day 2. Rats with transected spinal nerve roots had significantly more allodynia by 7 days, which was associated with IL- $1 \beta$ expression in dorsal and ventral horn astrocytes, and microglia activation. Repair of nerve roots with autologous intercostal nerve grafts and FGF in fibrin glue attenuated the allodynia, reduced IL-1 $\beta$ expression in astroctyes and reduced microglia activation, along with a significant increase in arginase I expression.

Conclusion: This study demonstrated a correlation between an increased number of IL-1 $\beta$-positive astrocytes and the development of allodynia. Our treatment significantly decreased IL-1 $\beta$-positive astrocytes, thus preventing the occurrence of neuropathic pain following multiple cervical root injuries.
\end{abstract}

\footnotetext{
${ }^{1}$ These authors contributed equally to this work.

*Corresponding author: Ming-Chao Huang, Department of Neurosurgery, Neurological Institute, Taipei Veterans General Hospital, No. 201, Shih-Pai Road, Sec. 2, Taipei 112, Taiwan. Tel.: +886 2 28757491; Fax: +886 2 28757588; E-mail: mchuang@vghtpe. gov.tw.
}

\section{Introduction}

Traumatic brachial plexus injury (BPI) occurs in about 4-9\% of all upper limb peripheral nerve injuries. Brachial plexus palsy can result from a difficult childbirth and is reported to occur in $0.1-0.4 \%$ of live births 
(Clarke and Curtis, 1995). Avulsion of one or more cervical nerve roots that forms the brachial plexus constitutes approximately $70 \%$ of severe BPI cases (Narakas, 1993). Injury may vary from axonotmesis to neurotmesis to the avulsion of rootlets from the spinal cord. Although there is no direct physical damage to the spinal cord, cervical root injuries are close to cell bodies and can lead to the dieback of spinal motor and dorsal root ganglion (DRG) neurons (Tandrup et al., 2000; Ma et al., 2001). Therefore, cervical root injuries are a combination of peripheral nerve and spinal cord injuries. We previously demonstrated, both in the laboratory and in clinical cases, that sensory and motor recoveries can be achieved by repairing transected cervical roots with intercostal nerve grafts and treating the repair site with acidic fibroblast growth factor (aFGF) and fibrin glue (Chuang et al., 2002; Hsu et al., 2004; Lee et al., 2004). However, in addition to loss of functions, the majority of patients with this type of injury suffer from excruciating pain. In some cases, the pain is so unbearable that patients would give up their existing motor function if it would cure the pain. Unfortunately, despite advances in pharmacology, surgery and physical therapies, there is currently no effective treatment for this type of neuropathic pain.

It is known that tissue injury results in the release of proinflammatory mediators (e.g., cytokines and chemokines) in the acute stage that, if not properly treated, can lead to chronic pain. In addition, some growth factors, such as nerve growth factor (NGF) and brain-derived neurotrophic factor (BDNF), have been shown to cause inflammatory reactions and lead to neuropathic pain (Ji et al., 1996; Obata et al., 2004). It is, therefore, crucial to evaluate whether the combination of nerve grafts and aFGF is beneficial or harmful for patients in terms of the development of neuropathic pain.

The results of our previous studies demonstrate that treatment with either aFGF or nerve grafts alone was not as effective as when these therapies were combined (Huang et al., 2007; Kuo et al., 2007; Lee et al., 2008). In the present study, all repair animals received the most effective treatment strategy (i.e., the combination of nerve grafting, aFGF and fibrin glue). Our previous data also showed that there was a limited time window for repair treatment. When the repair was done immediately after the injury, motor and sensory functions had significant recovery. The function can still be recovered when the repair treatment was applied 1 week after the injury. However, if the same repair was delayed for
3 weeks, there was no significant functional recovery despite some successful neuroregeneration (Huang et al, 2007, 2009). Since these data indicated that there might be significant difference in the spinal cord before and after the first week of injury, we chose to examine the effects of treatment on day 7.

\section{Materials and methods}

\subsection{Animals}

Adult female Sprague-Dawley rats $(250 \mathrm{~g})$ were randomly assigned to 3 treatment groups: sham (S), transection (T) or repair (R). Animals were housed in ventilated, humidity- and temperature-controlled rooms with a 12/12-h light/dark cycle. Food and water were provided ad libitum. Animals were maintained according to the Guide for the Care and Use of Laboratory Animals guidelines established by the Committee for the Care and Use of Laboratory Animals of the Institute of Laboratory Animal Resources Commission on Life Sciences, National Research Council, USA. The protocols were reviewed and approved by the Institutional Animal Care and Use Committee of Taipei Veterans General Hospital, Taiwan.

\subsection{Surgical procedure}

The surgical procedure was performed as reported previously (Huang et al., 2007). All animals were anesthetized with halothane $(1.5 \mathrm{v} / \mathrm{v}, 1.5 \mathrm{liters} / \mathrm{min}$ to maintain a breathing rate approximately 60 breaths/ min) and placed on a heating pad. Rectal temperatures were monitored during surgery and maintained at no less than $3^{\circ} \mathrm{C}$ below normal body temperature.

Animal in the sham group received a vertical incision to expose the spinous processes and laminae of the C2-T1 vertebrae. A left hemilaminectomy of the C5-7 vertebrae was performed. After the dura was opened, the incision was closed.

Animals in the transection group $(n=5)$ received a hemilaminectomy of the $\mathrm{C} 5-7$, as described in the sham group. The left C6-8 cervical roots were identified and the dura was opened. The C6-8 nerve roots were then gently pulled and cut where they exited from the cervical spinal cord; no proximal stump remained.

Animals in the repair group $(n=5)$ received the same transection procedure described for the transection group. Following transection of the left C6-8 
nerve roots, however, three intercostal nerves from T4-6 were harvested from the same animals and preserved in Hanks' solution. Intercostal nerves were cut transversely into two identical segments. Two grafts were used as autografts to repair one root: one graft for the ventral root and a second graft for the dorsal root. One end of the nerve graft was anastomosed to the severed nerve root with a 10-0 prolene suture, while the other end of the intercostal nerve was implanted into the spinal cord through a tiny incision in the pia mater. For the ventral root, grafts were inserted into the ventro-lateral aspect, whereas grafts for the dorsal root were inserted into the dorso-lateral aspect of the spinal cord. Following nerve root grafting, aFGF $(10 \mu \mathrm{g})$ mixed with fibrin glue was applied to the surgical area. The fibrin glue was prepared prior to the application by mixing the fibrinogen with an aprotinin solution. Aprotinin can stabilize fibrin by inhibiting fibrinolysis and therefore allows the slow release of aFGF from the glue cast. This solution was then mixed with thrombin containing calcium chloride and applied to the surgical area to form a glue cast.

\subsection{Mechanical sensitivity}

To quantify the mechanical sensitivity of the limb, von Frey filaments were used to test the frequency of foot withdrawal in response to mechanical stimuli on days 2 and 7 after surgery. The rats were placed on an elevated metal wire mesh floor in a small Lucite cubicle $(20 \times 8 \times 8 \mathrm{~cm})$, and $2 \mathrm{~g}$ of von Frey filaments were applied from underneath the wire mesh to the plantar surface of the ipsilateral forelimbs. The frequency of response out of a total of 10 stimuli was recorded. Evaluations were performed by personnel who were blinded to the treatment group of the animal.

\subsection{Immunohistostaining}

One week after the operation, animals were sacrificed and perfused with $4 \%$ paraformaldehyde. Cryosections $(10 \mu \mathrm{m})$ were stained using standard immunohistochemistry methods. Briefly, sections were blocked with $2 \%$ normal donkey serum (Jackson Immuno-Research, West Grove, PA) and incubated with the following primary antibodies: mouse anti-OX42 ( $1: 100$ dilution, Serotec), mouse anti-ED1 (1:300, Serotec), goat anti-IBA1 (1:200, Abcam), rabbit antiGFAP (1:500 dilution, Chemicon, Temecula, CA), goat anti-IL-1 $\beta$ ( $1: 500$ dilution, R\&D), or goat anti- arginase I ( $1: 250$ dilution, Santa Cruz). The following fluorescent secondary antibodies were then applied: Alexa 568 donkey anti-rabbit, Alexa 488 donkey antigoat, Cy3 donkey anti-goat, and Alexa 488 donkey anti-mouse (Jackson Immuno-Research, West Grove, PA). Sections were visualized at $200 \times$ magnification under a Zeiss light (Axioplan 2, Carl Zeiss) or fluorescent microscope (Axioskop 2, Carl Zeiss). One or two sections were selected randomly from each corresponding spinal cord level and a total of 5 sections were selected from each animal. All immunoreactive cells within dorsal horns and ventral horns were counted.

\subsection{Statistical analysis}

The data are expressed as the means \pm SD. All data obtained from the mechanical sensitivity and immunohistochemistry experiments were analyzed by one-way ANOVA for multiple group comparisons. Post-hoc analyses were performed with Tukey's multiple comparisons test of the means. Significance was assumed at $p<0.05$.

\section{Results}

\subsection{Allodynia behavior}

On day 2 after the operation, the results of the mechanical sensitivity test did not differ between treatment groups. On day 7, however, pain sensitivity to mechanical stimuli was significantly increased only in the transection group (Fig. 1).

\subsection{IL-1 $\beta$ expression following nerve root injury}

The number of IL-1 $\beta$ positive cells was significantly higher in the ipsilateral dorsal and ventral horns of the transection group when compared to the repair group $(p<0.05)$. There was no colocalization of IL-1 $\beta$ with either microglia stained with IBA1 or macrophages stained with ED1. However, a large proportion of IL-1 $\beta$ expression was associated with astrocytes stained with GFAP (Fig. 2). IL-1 $\beta$ expression was also found in motor neurons, but there were no significant differences among groups (data not shown).

\subsection{Astrocyte response to nerve root injury}

On the ipsilateral side, the number of astrocytes was increased in both the dorsal and ventral horns of the 


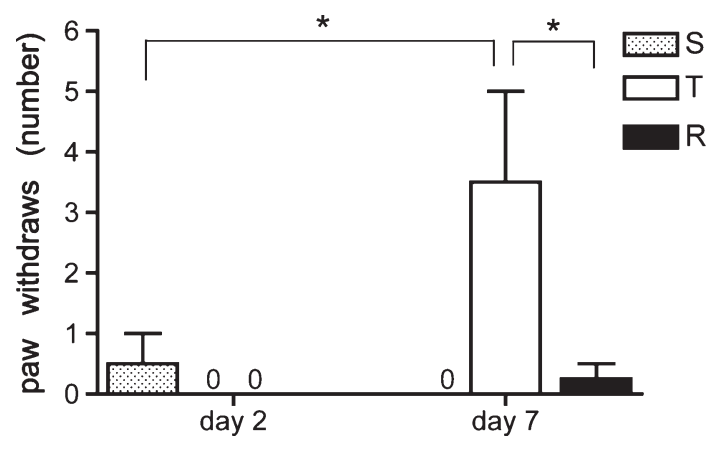

Fig. 1. Animal responses to the mechanical stimulation. Average mechanical allodynia data in the ipsilateral forepaw for sham (S), transection (T) and repair (R) groups after testing with von Frey filaments are presented. On day 2 , there was a mild response in some of the sham group animals; no response was observed in the other treatment groups. These differences were not statistically significant. By day 7 , the transection group showed significantly greater allodynia when compared to the repair group. No allodynia was observed in the sham group. Values are expressed as the mean $\pm \mathrm{SD} . *$ Indicates $p<0.05$.

spinal cord for the transection and repair groups but not the sham group. There was no difference in the total number of astrocytes between the transection and repair groups; however, the number of astrocytes with
IL-1 $\beta$-positive staining was significantly higher in the transection group when compared to the repair group (Fig. 3). On the contralateral side of the spinal cord, astrocytes activation was reduced in all three treatment groups.

\subsection{Microglial response to nerve root injury}

Significant microglial activation was only found in the ipsilateral dorsal and ventral horns of the transection group. There was a mild reaction in the repair group and almost no reaction in the sham group. There was no significant difference in microglial activation between the dorsal and ventral horns. Microglial activation on the contralateral side was approximately $50 \%$ less than that observed on the ipsilateral side (Fig. 4).

\subsection{Macrophages and motor neurons express Arginase I}

In the repair group, we observed many Arginase I (Arg I)-positive macrophages in the ipsilateral dorsal root entry zone with abundant translucent cytoplasm and a phagocytic appearance (Fig. 5, arrowhead). No Arg I-positive macrophages were seen in the transec-
(A)
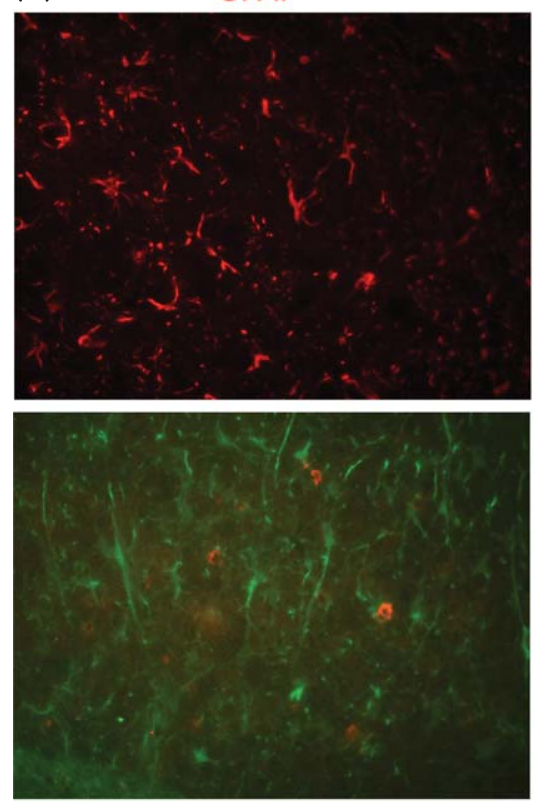

IL1 $\beta+E D 1$
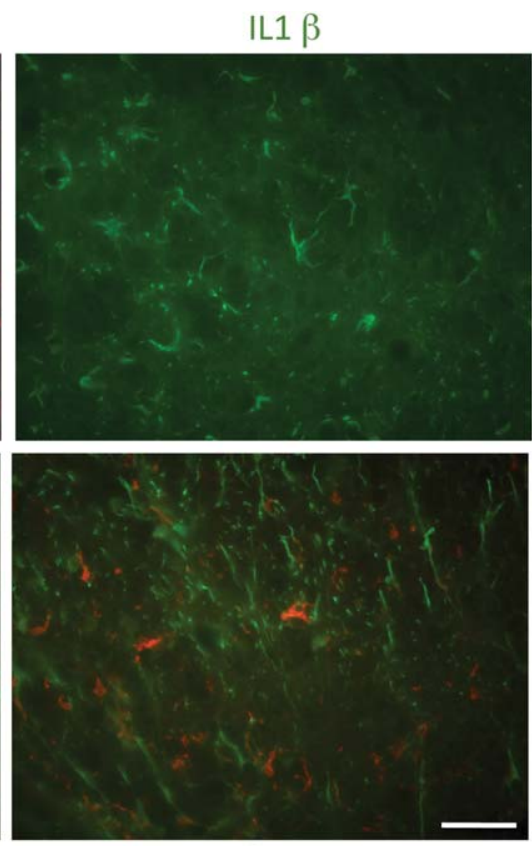

IL1 $\beta+\mid B A 1$

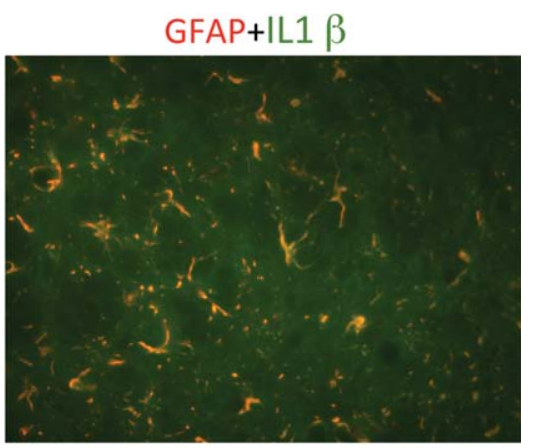

(B)

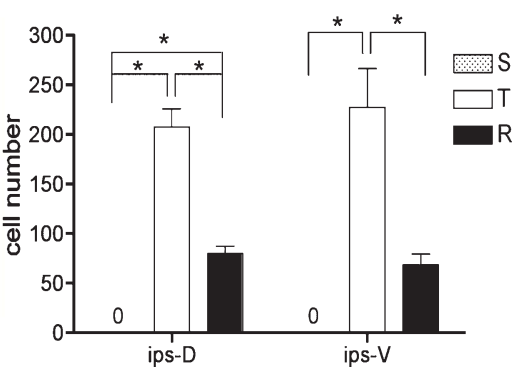

Fig. 2. (A) Representative images demonstrating co-labeling of IL-1 $\beta$ with GFAP and lack of co-localization of IL- $1 \beta$ with ED1 and IBA1 on day 7 after surgery. For all images, the scale bar is equal to $100 \mu \mathrm{m}$. (B) Quantitative measurements of IL-1 $\beta$ positive cells. Ips-D: ipsilateral dorsal horn; Ips-V: ipsilateral ventral horn. Values are expressed as the mean \pm SD. * Indicates $p<0.05$. 

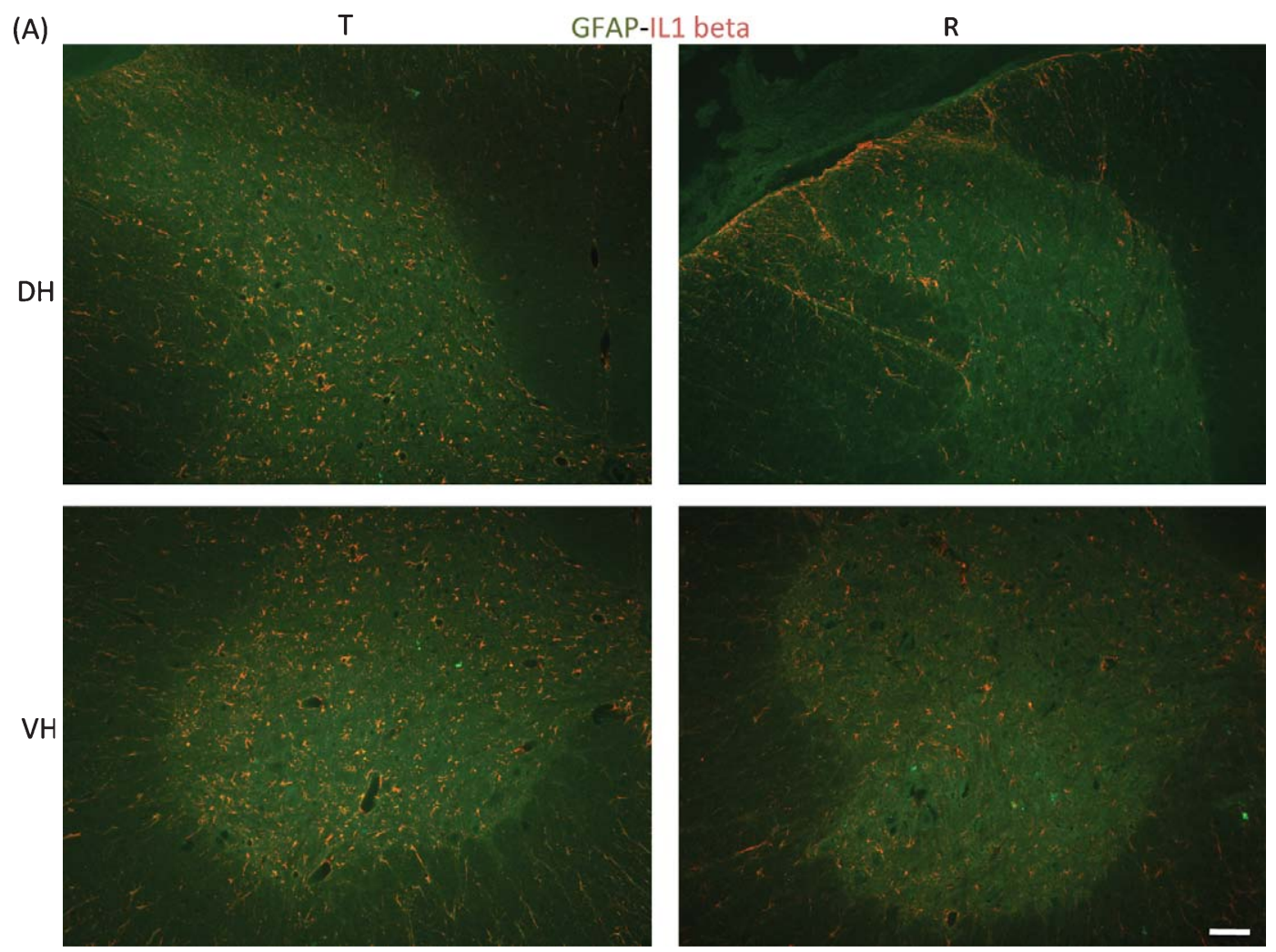

(B)

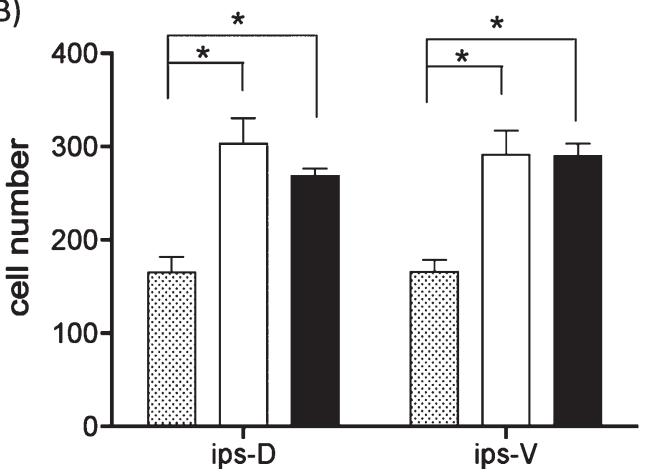

(C)

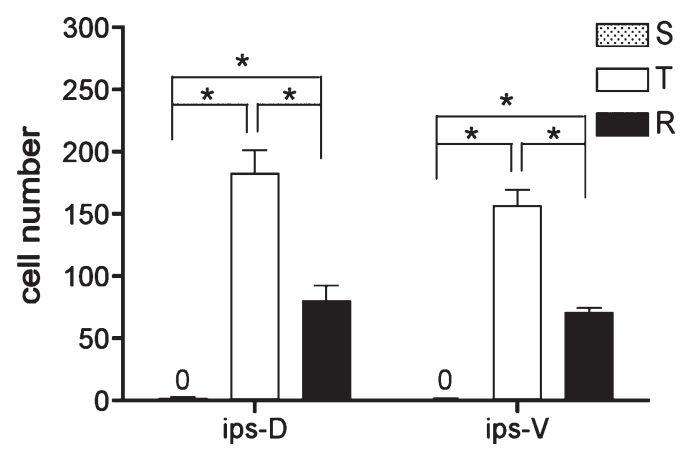

Fig. 3. (A) Representative ipsilateral spinal cord sections showing the dorsal horn (DH) and ventral horn (VH) stained against GFAP and IL-1 $\beta$ on day 7 after surgery. For all images, the scale bar is equal to $100 \mu \mathrm{m}$. (B) Quantitative measurements of GFAP-positive cells. (C) Quantitative measurements of cells with co-expression of GFAP and IL-1 $\beta$. Values are expressed as the mean \pm SD. * Indicates $p<0.05$.

tion or sham group. Although many neurons in the ipsilateral ventral horn were Arg I-positive in the repair group, very few Arg I-positive neurons were found in the transection group. There was a significant difference in the number of Arg I-positive macrophages and neurons between the repair group and either the sham or transection group.

\section{Discussion}

In the present study, we compared the inflammatory status of animals with C6-8 root transections on day 7 after surgery with or without surgical repair by a combination of aFGF and nerve grafts. Transections that were repaired with aFGF and nerve grafts showed 
(A)
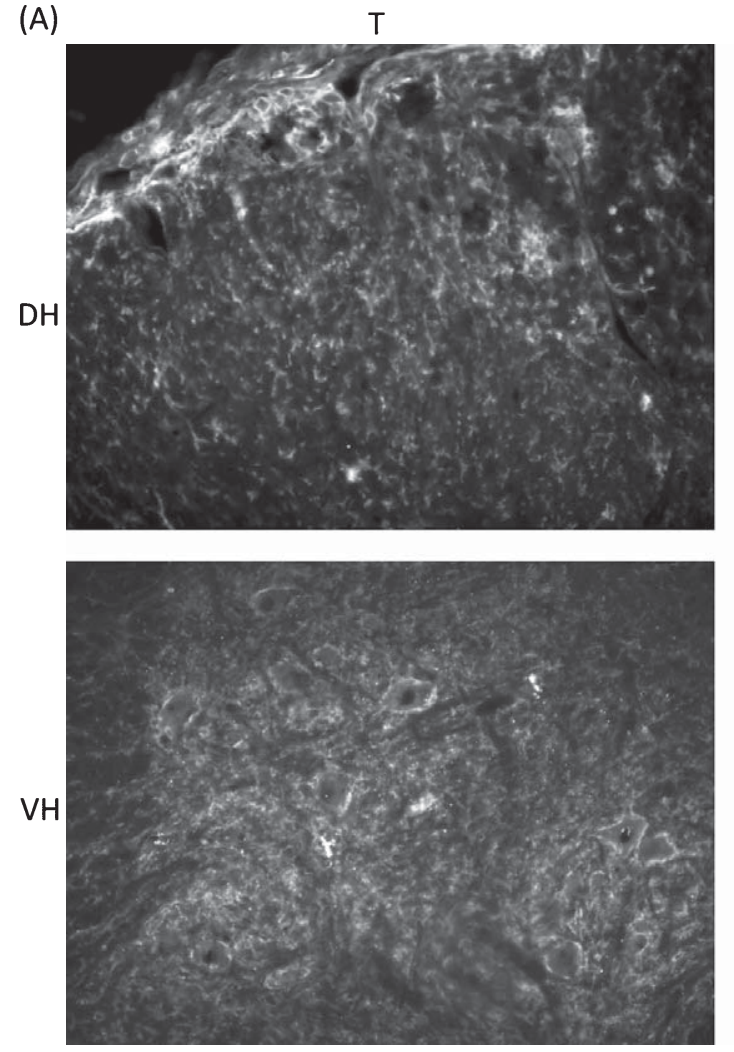

(B)

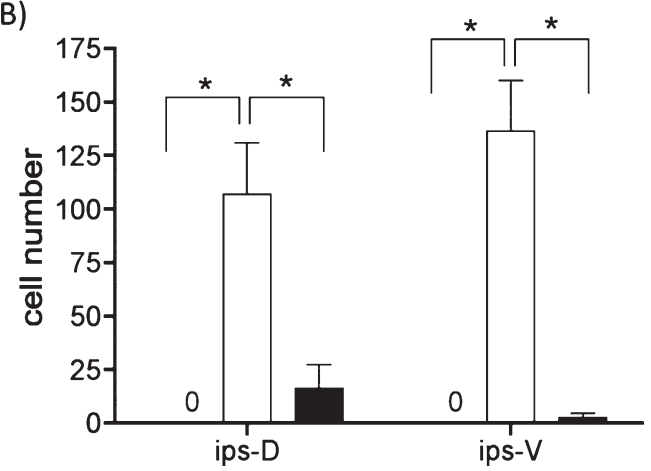

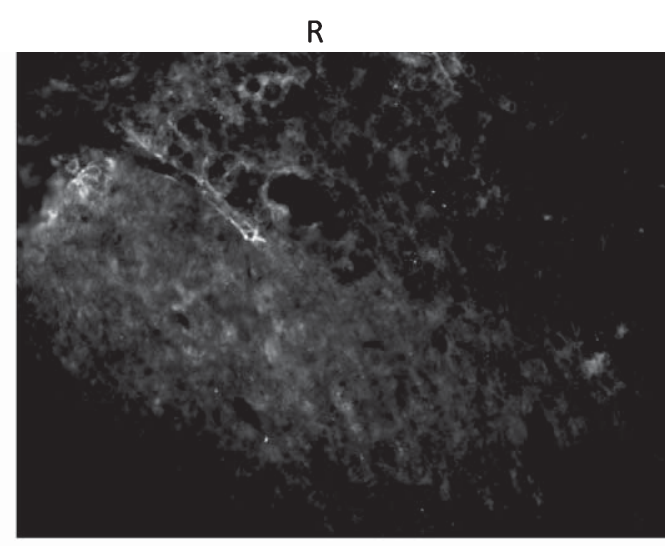

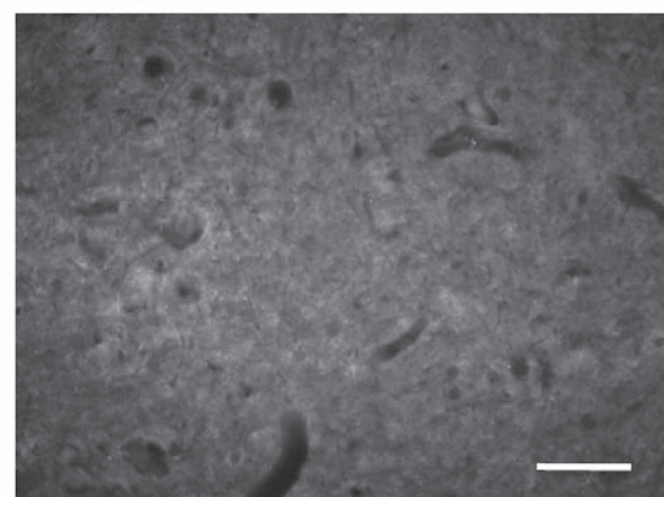

(C)

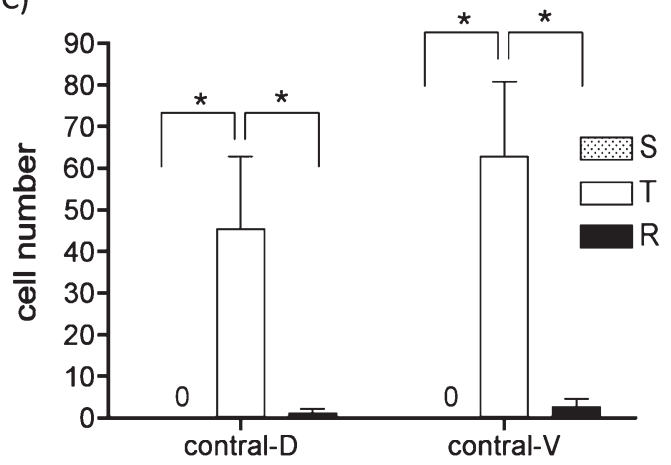

Fig. 4. Microglial activation in the spinal cord on day 7 after surgery. (A) OX-42 staining shows more intense microglial activation in the T group when compared to the $\mathrm{R}$ group. For all images, the scale bar is equal to $100 \mu \mathrm{m}$. (B) Quantitative measurements of activated microglia on the ipsilateral side of the spinal cord. (C) Quantitative measurements of activated microglia on the contralateral side of the spinal cord. Contral-D: contralateral dorsal horn; Contral-V: contralateral ventral horn. Values are expressed as the mean $\pm \mathrm{SD}$. ${ }^{*}$ Indicates $p<0.05$.

significantly decreased microglial and IL-1 $\beta$-positive astrocyte reactions in the spinal cord. The repair strategy was also found to upregulate Arg I, an enzyme that favors the differentiation of macrophages toward an M2 phenotype and increases neuronal survival. As a result, animals in the repair group demonstrated less severe allodynia than those in the transection group.
In the transection group, allodynia was absent on day 2 but occurred on day 7 post-injury. This allodynia behavior was different than is seen with a single root transection at $\mathrm{C} 7$. With this type of injury, transient allodynia is observed at day 1 but disappears by day 7 post-surgery (Rothman et al., 2005). These differences in allodynia onset in the single root injury 
(A)
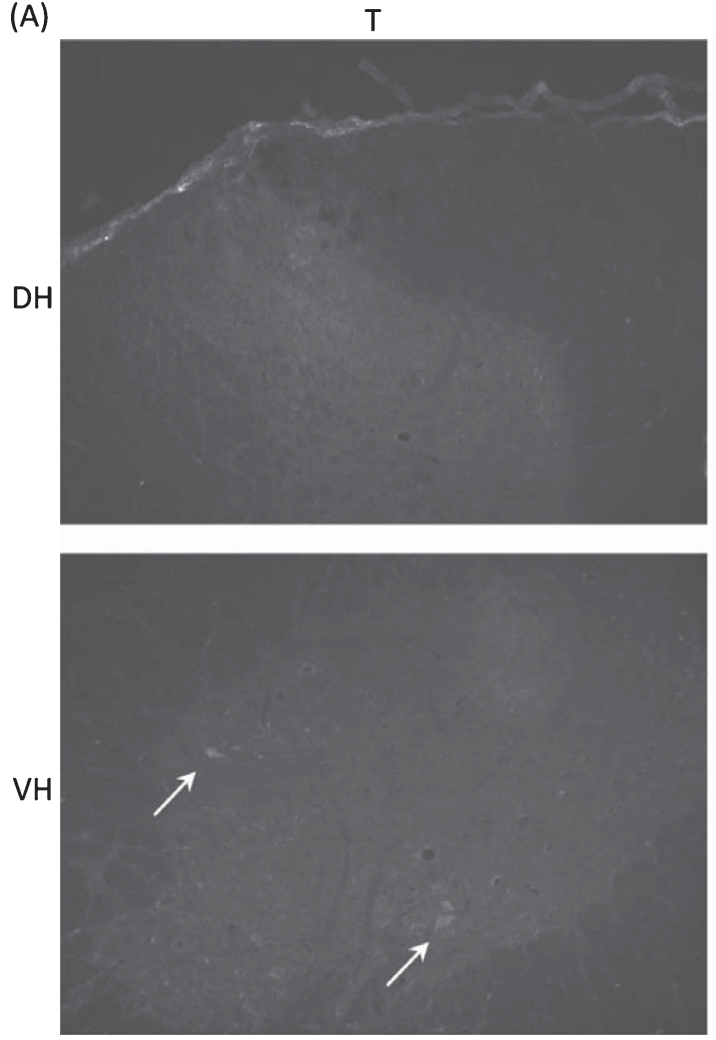

(B)

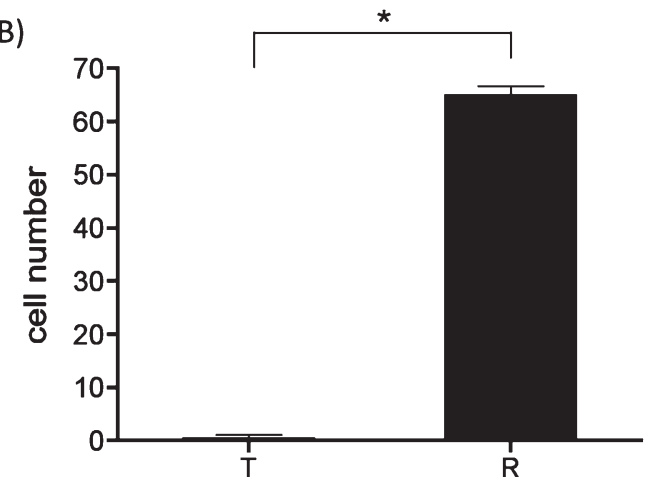

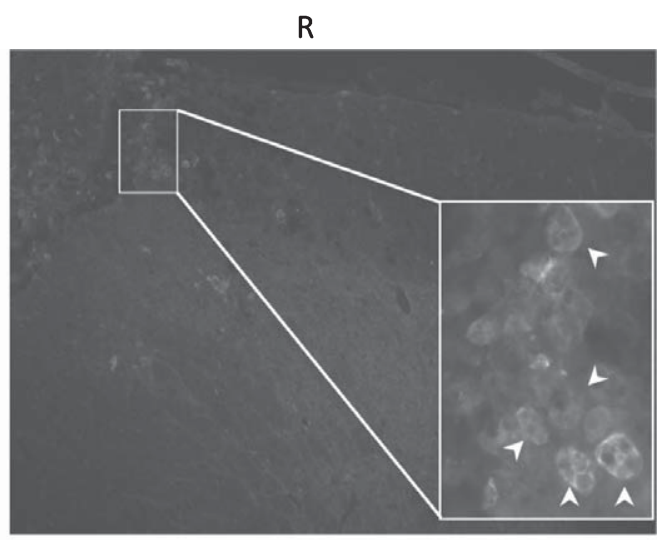

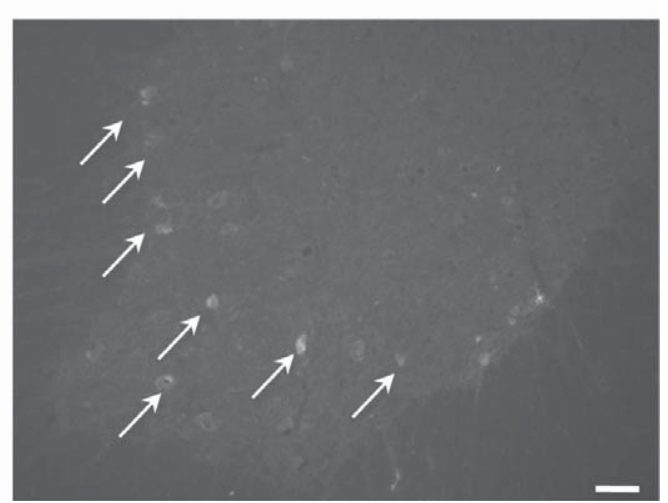

(C)

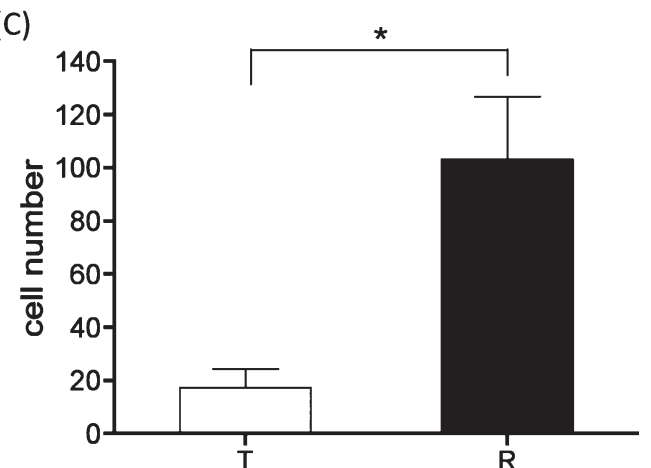

Fig. 5. Arginase I expression on day 7 after surgery. (A) In the DREZ, many Arg I-positive macrophages (arrowheads) are present in the R group, but very few are found in the T group. In the VH, there are more Arg I-positive motorneurons (arrows) in the R group than in the T group. For all images, the scale bar is equal to $100 \mu \mathrm{m}$. (B) Quantitative measurements of Arg-I-positive macrophages in the DREZ. (C) Quantitative measurements of Arg I-positive motorneurons in the VH. Values are expressed as the mean \pm SD. $*$ indicates $p<0.05$.

model may be due to the presence of impulse transmission from neighboring afferents. In the present model, all roots responsible for the transmission of sensation in the forepaw were severed; therefore, no allodynia could occur at the early time point. In contrast, a single root transection at $\mathrm{C} 7$ is not sufficient to obliterate forepaw sensation because the $\mathrm{C} 6$ and $\mathrm{C} 8$ nerve roots remain intact, allowing the transmission of pain sensations. Similarly, allodynia is also observed in L5 root compression or L5 ligation models as early as 1 day after injury (Hashizume et al., 2000; Winkelstein et al., 2001; Winkelstein and DeLeo, 2002), because sensory information can be transmitted through the neighboring L4 and L6 afferents. 
To date, a number of treatment options have been tested to control neuropathic pain by targeting glia or cytokines. Therapies that specifically target microglia, such as minocycline (Rothman et al., 2009), or antagonists, such as sTNFR and IL-1 ra (Sweitzer et al., 2001), are able to attenuate allodynia but fail to abolish spinal microglial activation and proliferation. In contrast, in this study, we have shown that nerve grafts combined with the application of aFGF effectively decreases microglia activation. Given that microglial activation is recognized as an early injury response, the decrease in the number of microglia in the repair group may indicate that our treatment limits the extent of acute inflammation.

In addition to decreasing microglial activation, our treatment reduced IL-1 $\beta$ expression. IL- $1 \beta$ is secreted under conditions associated with pain (Watkins et al., 1995; Milligan et al., 2003), and levels are known to increase continuously for at least 35 days in response to sciatic nerve injury (DeLeo et al., 1997). IL-1 $\beta$ is also elevated in the cerebral spinal fluid of patients suffering from chronic pain (Alexander et al., 2005). In this study, we showed that astrocytes are involved in the maintenance of IL- $1 \beta$ expression. The length of astrocyte activation has been shown to facilitate the development of neuropathic pain, especially when astrocytes are actively producing IL-1 $\beta$ (Wang et al., 2009). Therefore, the decrease of IL-1 $\beta$ expression in astrocytes may decrease the occurrence of allodynia following cervical root transection injuries.

In our combined therapy, the nerve graft served as a nerve conduit to guide the regeneration of sensory axons into the spinal cord. These nerve grafts also provided a rich source of Schwann cells that are known to promote axon growth and nerve regeneration (Hall, 1986; Torigoe et al., 1996; Hoke et al., 2002). Thus, nerve grafts aided in the reconstruction of normal axonal circuits in the dorsal horns. Derangement of sensory fibers in the dorsal horn is known to play a crucial role in the development of allodynia; therefore, the combined therapy used here is likely to decrease painful sensations.

We also observed a significant increase in the number of Arg I-positive M2 macrophages, in the repair group. Because the upregulation of Arg I limits nitrite oxide production (Chang et al., 1998), Arg I expression may downregulate inflammation. We also observed a significant increase in the number of Arg I-positive neurons in the repair group. Arg I has been shown to inhibit neuronal apoptosis (Esch et al., 1998), pre- vent neuronal dieback, and promote neuronal survival. Recently, we found that aFGF induced Th2 cytokine IL-4 expression and a sequential M2 marker of Arg I upregulation (Kuo et al., 2011). Arg I can also upregulate the synthesis of polyamines that promote axonal regeneration in an inhibitory extracellular environment after injury (Gilad and Gilad, 1988; Cai et al., 2002). Thus, the upregulation of Arg I after treatment may decrease inflammatory response, enhance neuronal survival, and encourage regeneration of sensory axons through the dorsal root entry zone, an inhibitory environment, into the dorsal horn and lead to the reestablishment of sensory circuits in the spinal cord. The decrease in inflammation and the reestablishment of sensory circuits can, in turn, decrease the occurrence of neuropathic pain.

The combination of nerve graft and aFGF might play a crucial role in maintaining Schwann cell-axon interactions. A recent study by Furusho et al. demonstrated that FGF signaling in non-myelinating Schwann cells is essential in maintaining the integrity of unmyelinated sensory axon. The disruption of FGF signaling resulted in sensory axon neuropathy and thermal pain sensitivity (Furusho et al., 2009). Therefore, providing aFGF exogenously along with nerve grafts might be helpful in maintaining FGF signals and then beneficial for enhancing axon regeneration and decreasing pain sensation.

In conclusion, treatment of cervical root transection injuries with a combination of nerve grafts, aFGF, and fibrin glue can lead to decreased occurrence of allodynia and recovery of motor control and sensation. Although not conclusive, our study indicates that neuropathic pain caused by severe BPI maybe ameliorated by proper treatment in the acute stage following injury.

\section{Author disclosure statement}

The authors declare no competing financial interests.

\section{Acknowledgments}

The authors would like to thank Shao-En Chang, Kai-Ting Chang and Ching-Jung Chen for their technical assistance. This study was supported by the following grants: NSC 95-2314-B-075-067, NSC 992314-B-075-010-MY3, VWY-96-001, VWY-98-001, V95S6-001, V96S6-001, and V97S6-001. 


\section{References}

Alexander, G.M., van Rijn, M.A., van Hilten, J.J., Perreault, M.J. \& Schwartzman, R.J. (2005). Changes in cerebrospinal fluid levels of pro-inflammatory cytokines in CRPS. Pain, 116(3), 213-219.

Cai, D., Deng, K., Mellado, W., Lee, J., Ratan, R.R. \& Filbin, M.T. (2002). Arginase I and polyamines act downstream from cyclic AMP in overcoming inhibition of axonal growth MAG and myelin in vitro. Neuron, 35(4), 711-719.

Chang, C.I., Liao, J.C. \& Kuo, L. (1998). Arginase modulates nitric oxide production in activated macrophages. Am J Physiol, 274(1 Pt 2), H342-H348.

Chuang, T.Y., Huang, M.C., Chen, K.C., Chang, Y.C., Yen, Y.S., Lee, L.S. \& Cheng, H. (2002). Forelimb muscle activity following nerve graft repair of ventral roots in the rat cervical spinal cord. Life Sci, 71(5), 487-496.

Clarke, H.M. \& Curtis, C.G. (1995). An approach to obstetrical brachial plexus injuries. Hand Clin, 11(4), 563-580; discussion 580-561.

DeLeo, J.A., Colburn, R.W. \& Rickman, A.J. (1997). Cytokine and growth factor immunohistochemical spinal profiles in two animal models of mononeuropathy. Brain Resi, 759(1), 50-57.

Esch, F., Lin, K.I., Hills, A., Zaman, K., Baraban, J.M., Chatterjee, S., Rubin, L., Ash, D.E. \& Ratan, R.R. (1998). Purification of a multipotent antideath activity from bovine liver and its identification as arginase: nitric oxide-independent inhibition of neuronal apoptosis. J Neurosci, 18(11), 4083-4095.

Furusho, M., Dupree, J.L., Bryant, M. \& Bansal, R. (2009). Disruption of fibroblast growth factor receptor signaling in nonmyelinating Schwann cells causes sensory axonal neuropathy and impairment of thermal pain sensitivity. J Neurosci, 29(6), 1608-1614.

Giacobini, M.M., Hoffer, B.J., Zerbe, G. \& Olson, L. (1991). Acidic and basic fibroblast growth factors augment growth of fetal brain tissue grafts. Exp Brain Res, 86(1), 73-81.

Gilad, G.M. \& Gilad, V.H. (1988). Early polyamine treatment enhances survival of sympathetic neurons after postnatal axonal injury or immunosympathectomy. Brain Res, 466(2), 175-181.

Hall, S.M. (1986). Regeneration in cellular and acellular autografts in the peripheral nervous system. Neuropathol Appl Neurobiol, 12(1), 27-46.

Hashizume, H., DeLeo, J.A., Colburn, R.W. \& Weinstein, J.N. (2000). Spinal glial activation and cytokine expression after lumbar root injury in the rat. Spine, 25(10), 1206-1217.

Hoke, A., Gordon, T., Zochodne, D.W. \& Sulaiman, O.A. (2002). A decline in glial cell-line-derived neurotrophic factor expression is associated with impaired regeneration after long-term Schwann cell denervation. Exp Neurol, 173(1), 77-85.

Hsu, S.P., Shih, Y.H., Huang, M.C., Chuang, T.Y., Huang, W.C., Wu, H.M., Lin, P.H., Lee, L.S. \& Cheng, H. (2004). Repair of multiple cervical root avulsion with sural nerve graft. Injury, 35(9), 896-907.

Huang, M.C., Chang, P.T., Tsai, M.J., Kuo, H.S., Kuo, W.C., Lee, M.J., Lo, M.J., Lee, I.H., Huang, W.C., Lee, L.M., Shih, Y.H., Lee, L.S. \& Cheng, H. (2007). Sensory and motor recovery after repairing transected cervical roots. Surg Neurol, 68(5), S1: 17-24.

Huang, M.C., Lo, M.J., Lin, Y.L., Chang, S.E., Huang, W.C., Kuo, W.C., Tsai, M.J., Kuo, H.S., Shih, Y.H. \& Cheng, H. (2009). Functional recovery after the repair of transected cervical roots in the chronic stage of injury. J Neurotrauma, 26(10), 17951804.

Ji, R.R., Zhang, Q., Pettersson, R.F. \& Hokfelt, T. (1996). aFGF, bFGF and NGF differentially regulate neuropeptide expression in dorsal root ganglia after axotomy and induce autotomy. Regul Pept, 66(3), 179-189.

Kuo, H.S., Tsai, M.J., Huang, M.C., Chiu, C.W., Tsai, C.Y., Lee, M.J., Huang, W.C., Lin, Y.L., Kuo, W.C., Cheng, H. (2011). Acid fibroblast growth factor and peripheral nerve grafts regulate Th2 cytokine expression, macrophage activation, polyamine synthesis, and neurotrophin expression in transected rat spinal cords. J Neurosci, 31(11), 4137-4147.

Kuo, H.S., Tsai, M.J., Huang, M.C., Huang, W.C., Lee, M.J., Kuo, W.C., You, L.H., Szeto, K.C., Tsai, I.L., Chang, W.C., Chiu, C.W., Ma, H., Chak, K.F. \& Cheng, H. (2007). The combination of peripheral nerve grafts and acidic fibroblast growth factor enhances arginase I and polyamine spermine expression in transected rat spinal cords. Biochem Biophys Res Commun, 357(1), 1-7.

Lee, L.M., Huang, M.C., Chuang, T.Y., Lee, L.S., Cheng, H. \& Lee, I.H. (2004). Acidic FGF enhances functional regeneration of adult dorsal roots. Life Sci, 74(15), 1937-1943.

Lee, M.J., Chen, C.J., Cheng, C.H., Huang, W.C., Kuo, H.S., Wu, J.C., Tsai, M.J., Huang, M.C., Chang, W.C. \& Cheng, H. (2008). Combined treatment using peripheral nerve graft and FGF-1: changes to the glial environment and differential macrophage reaction in a complete transected spinal cord. Neurosci Lett, 433(3), 163-169.

Ma, J., Novikov, L.N., Wiberg, M. \& Kellerth, J.O. (2001). Delayed loss of spinal motoneurons after peripheral nerve injury in adult rats: a quantitative morphological study. Exp Brain Res, 139(2), 216-223.

Milligan, E.D., Twining, C., Chacur, M., Biedenkapp, J., O’Connor, K., Poole, S., Tracey, K., Martin, D., Maier, S.F. \& Watkins, L.R. (2003). Spinal glia and proinflammatory cytokines mediate mirror-image neuropathic pain in rats. J Neurosci, 23(3), 10261040.

Narakas, A.O. (1993). Lesions found when operating traction injuries of the brachial plexus. Clin Neurol Neurosurg, 95 Suppl, S56-S64.

Nishi, R. (1994). Neurotrophic factors: two are better than one. Science (New York, NY), 265(5175), 1052-1053.

Obata, K., Yamanaka, H., Dai, Y., Mizushima, T., Fukuoka, T., Tokunaga, A., Yoshikawa, H. \& Noguchi, K. (2004). Contribution of degeneration of motor and sensory fibers to pain behavior and the changes in neurotrophic factors in rat dorsal root ganglion. Exp Neurol, 188(1), 149-160.

Rothman, S.M., Guarino, B.B. \& Winkelstein, B.A. (2009). Spinal microglial proliferation is evident in a rat model of painful disc herniation both in the presence of behavioral hypersensitivity and following minocycline treatment sufficient to attenuate allodynia. J Neurosci Res, 87(12), 2709-2717. 
Rothman, S.M., Kreider, R.A. \& Winkelstein, B.A. (2005). Spinal neuropeptide responses in persistent and transient pain following cervical nerve root injury. Spine (Phila Pa 1976), 30(22), 2491-2496.

Sweitzer, S., Martin, D. \& DeLeo, J.A. (2001). Intrathecal interleukin-1 receptor antagonist in combination with soluble tumor necrosis factor receptor exhibits an anti-allodynic action in a rat model of neuropathic pain. Neuroscience, 103(2), 529539.

Tandrup, T., Woolf, C.J. \& Coggeshall, R.E. (2000). Delayed loss of small dorsal root ganglion cells after transection of the rat sciatic nerve. J Comp Neurol, 422(2), 172-180.

Torigoe, K., Tanaka, H.F., Takahashi, A., Awaya, A. \& Hashimoto, K. (1996). Basic behavior of migratory Schwann cells in peripheral nerve regeneration. Exp Neurol, 137(2), 301-308.

Wang, W., Mei, X., Huang, J., Wei, Y., Wang, Y., Wu, S. \& Li, Y. (2009). Crosstalk between spinal astrocytes and neurons in nerve injury-induced neuropathic pain. PLoS One, 4(9), e6973.
Watkins, L.R., Maier, S.F. \& Goehler, L.E. (1995). Immune activation: the role of pro-inflammatory cytokines in inflammation, illness responses and pathological pain states. Pain, 63(3), 289302.

Winkelstein, B.A. \& DeLeo, J.A. (2002). Nerve root injury severity differentially modulates spinal glial activation in a rat lumbar radiculopathy model: considerations for persistent pain. Brain Res, 956(2), 294-301.

Winkelstein, B.A., Rutkowski, M.D., Sweitzer, S.M., Pahl, J.L. \& DeLeo, J.A. (2001). Nerve injury proximal or distal to the DRG induces similar spinal glial activation and selective cytokine expression but differential behavioral responses to pharmacologic treatment. J Comp Neurol, 439(2), 127-139. 